\title{
Growth and characterization of pure and lithium doped strontium tartrate tetrahydrate crystals by solution-gel technique
}

\author{
B SURESH KUMAR, M H RAHIM KUTTY, M R SUDARSANA KUMAR ${ }^{\dagger}$ and \\ K RAJENDRA BABU* \\ Department of Physics, ${ }^{\dagger}$ Department of Chemistry, Mahatma Gandhi College, Thiruvananthapuram 695 004, India
}

MS received 19 May 2006; revised 29 June 2007

\begin{abstract}
The effect of lithium ion as dopant on the size and transparency of strontium tartrate tetrahydrate $\left(\mathrm{SrC}_{4} \mathrm{H}_{4} \mathrm{O}_{6} \cdot 4 \mathrm{H}_{2} \mathrm{O}\right)$ crystals are presented in this paper. Growth of single crystals of undoped and lithium doped strontium tartrate tetrahydrate by controlled diffusion of strontium nitrate into the gel charged with tartaric acid at room temperature are narrated. The lithium ion enhances the size and transparency of the doped crystals. The crystal structure of the compound was confirmed by X-ray diffractometry and dopant concentration with ICP-atomic emission spectrometer system. Thermal decomposition of the grown crystals is investigated by TGA and DTA studies. The FTIR spectra of pure and doped crystals are recorded and analysed. Kurtz powder technique has been used to test SHG efficiency of the crystals.
\end{abstract}

Keywords. Strontium tartrate tetrahydrate crystals; lithium ion; crystal growth; solution-gel technique.

\section{Introduction}

Single crystals are the backbone of the modern technological revolution. The impact of single crystals is clearly visible in industries like semiconductor, optics, etc. With the invention of lasers, the field of non-linear optics touched new heights and practical implementation was possible with the application of non-linear optical crystals. Growth of single crystals of strontium tartrate tetrahydrate (SrTTH) and trihydrate has attracted many researchers (Patel and Arora 1976, 1977; Arora et al 2004a, b, c, 2005a, b) on account of their ferroelectric and non-linear optical characteristics based on their second harmonic generation (SHG). Now-a-days great attention has been devoted to the growth and characterization of pure and doped tartrate crystals with the aim of identifying new materials for practical purposes.

The effect of dopants on various properties of single crystals are of great interest from both solid state science as well as technological points of view. Additions of dopants have a profound influence on the growth kinetics, morphology and second harmonic efficiency of tartrate crystals. The crystals of strontium tartrate tetrahydrate grown in silica gel medium in pure form and doped with chromium, iron, cobalt, nickel and cadmium have already been reported (Dennis and Henisch 1967; Rethinam et al 1993, $1994 a, b)$. The growth of vanadyl doped strontium tartrate tetrahydrate crystals (Satyanarayana et al 1985) lead doped strontium tartrate tetrahydrate crystals (Rahimkutty et al

*Author for correspondence (mgkrb1956@yahoo.co.in)
2001) and copper and barium added strontium tartrate trihydrate have also been reported (Viji et al 2005). But in the literature survey, to the best of our knowledge, there is no report on the systematic growth and characterization of lithium doped strontium tartrate tetrahydrate [SrTLiTH] crystals. In the present work, we have attempted to grow pure and lithium doped strontium tartrate tetrahydrate crystals by gel technique. Unlike the conventional growth of SrTTH crystals in the gel medium, SrTLiTH crystals were grown on the surface of the gel. This growth experiment yielded crystals over the gel surface similar to the growth of $p$-chloroacetanilide crystals which were already reported (Rajendra Babu et al 1997) by using solution-gel technique. The grown crystals were characterized by Xray diffraction technique, AES study, FTIR analysis, TGA, DTA and NLO studies.

\section{Experimental}

\subsection{Crystal growth}

Most of the tartrate compounds are insoluble in water and decompose before melting. Hence, single crystals of such type of compounds cannot be grown by either slow evaporation or melt technique, but can be grown by silica gel method. A test tube diffusion method (Henisch 1973) was employed to grow pure and lithium doped strontium tartrate tetrahydrate crystals in the gel medium. The AR grade chemicals (MERCK) were used to grow the crystals. The crystallization apparatus employed was corning glass test tube $(25 \mathrm{~mm}$ diameter and $200 \mathrm{~mm}$ length). Gels were 
prepared by mixing sodium meta silicate solution of appropriate specific gravity and one molar solution of tartaric acid so that the desired $\mathrm{pH}$ of the mixture could be obtained. The specific gravity and $\mathrm{pH}$ were varied between $1.02 \mathrm{~g} / \mathrm{cc}$ and $1.05 \mathrm{~g} / \mathrm{cc}$ and 5 and 7 , respectively. After mixing, the solution was allowed to set for about $24 \mathrm{~h}$. Over the set gel, 1 molar strontium nitrate solution was gently poured with the help of a pipette, so as to allow the solution to fall steadily along the walls of the tube without disturbing the gel surface. The open end of the test tube was closed with transparent sheet of plastic to avoid evaporation of the solution and contamination of impurities. The test tubes were kept undisturbed at room temperature. To grow doped crystals, an aqueous solution of lithium nitrate of varying concentrations, $0 \cdot 01-0 \cdot 1 \mathrm{M}$, was mixed with the top solution.

\subsection{AES and XRD studies}

The impurity concentration of the dopant was determined by using inductively coupled plasma atomic emission spectrometer (ICPAES) of Thermo Electron Corporation, model IRIS INTREPID II XSP of detection limit ppb level. The X-ray powder diffraction pattern of lithium doped strontium tartrate crystals was obtained using high resolution BRUKER, AXS D8 advance model X-ray diffractometer with $\mathrm{CuK} \alpha 1(\lambda=1.54056 \AA)$ radiation in the $2 \theta$ range, $10-60^{\circ}$, the scanning speed of the specimen being $2 \% \mathrm{~min}$. In the present study, X-ray powder pattern of lithium doped strontium tartrate grown on gel surface was obtained and used to identify the grown material.

\subsection{Thermal studies}

The thermal decomposition behaviour of grown crystals was studied by thermogravimetric analysis (TGA) and differential thermal analysis (DTA). The TGA was carried out from room temperature to $800^{\circ} \mathrm{C}$ at a heating rate of $10^{\circ} \mathrm{C} / \mathrm{min}$ in an atmosphere of air by using METTLER TOLEDO S R SYSTEM.

\subsection{FTIR spectrum}

The FTIR spectrum of lithium doped strontium tartrate tetrahydrate crystals was recorded using Thermo-Nicolet Avtar 370 FTIR spectrometer using $\mathrm{KBr}$ pellet technique in the wavelength range $400-4000 \mathrm{~cm}^{-1}$.

\subsection{Non-linear optical studies}

Kurtz and Perry powder second harmonic generation (SHG) method was used to sense NLO behaviour of crystals. To measure SHG efficiency, samples were derived from the crystals grown with full morphology, which ensures homogeneity of the material. The input power of the laser beam was measured to be $5.7 \mathrm{mj} /$ pulse. The sample was tested using the high intensity $\mathrm{Nd}-\mathrm{YAG}$ laser $(\lambda=$ $1064 \mathrm{~nm})$ at the Department of Inorganic and Physical Chemistry, Indian Institute of Science, Bangalore.

\section{Results and discussion}

\subsection{Crystal growth}

The strontium nitrate diffused through the gel and white thin film appeared just below the gel surface. Tiny crystals were first sighted in about $24 \mathrm{~h}$. The size and number of isolated crystals increased in about 10 days. Highly transparent crystals with good morphological perfection appeared $1 \mathrm{~cm}$ below the gel-solution interface within two weeks. The pure strontium tartrate crystals were harvested after a month. The optimum conditions to grow the best quality strontium tartrate single crystals were at $\mathrm{pH}$ 5-6, gel density, $1.04 \mathrm{~g} / \mathrm{cc}$ and growth temperature, at about $30^{\circ} \mathrm{C}$. A change in the $\mathrm{pH}$ of the gel solution other than this range resulted in poor quality crystals. The
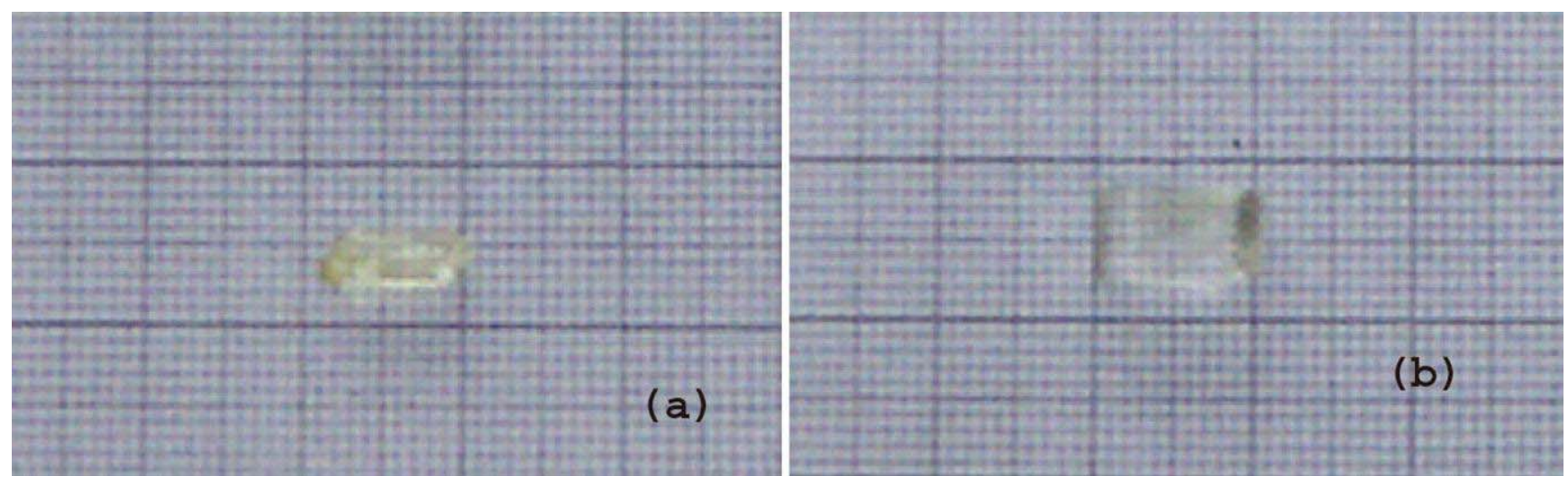

Figure 1. (a) Pure SrTTH and (b) lithium doped SrTTH crystals. 
Table 1. Effect of parameters on the growth of pure $\mathrm{SrTTH}\left(\mathrm{C}_{4} \mathrm{H}_{4} \mathrm{O}_{6} \mathrm{Sr} \cdot 4 \mathrm{H}_{2} \mathrm{O}\right.$ ) crystals. (One parameter is varied and other, kept constant).

\begin{tabular}{lc}
\hline Variation in gel density, & Nucleation density is maximum at $1.03 \mathrm{~g} / \mathrm{cc}$ and size of the \\
$1.02,1.03,1.04$ and $1.05 \mathrm{~g} / \mathrm{cc}$ & crystal is maximum at $1.04 \mathrm{~g} / \mathrm{cc}$ with minimum number of crystals. \\
Variation in gel pH, & Nucleation density is maximum at 6.5 and \\
$5,5 \cdot 5,6,6 \cdot 5,7$ & minimum at 5 . Size of the crystal is maximum at 5.5. \\
\hline
\end{tabular}

Table 2. Effect of dopant concentration on the growth of lithium doped SrTTH crystals (1 M strontium nitrate solution, $1 \mathrm{M}$ tartaric acid, gel density $1.04 \mathrm{~g} / \mathrm{cc}, \mathrm{pH} 6.5$ ).

\begin{tabular}{lcl}
\hline $\begin{array}{l}\text { Concentration of } \\
\text { lithium nitrate solution }\end{array}$ & Size of the crystals $\left(\mathrm{mm}^{3}\right)$ & Transparency of the crystals \\
\hline $0.01 \mathrm{M}$ & $(1.5-2) \times 1 \times 1$ & Pale yellow in colour \\
$0.04 \mathrm{M}$ & $(2-2.7) \times 1.5 \times 3$ & Semi transparent crystals \\
$0.07 \mathrm{M}$ & $(5-7) \times 3 \times 3$ & Transparent crystals \\
$0.1 \mathrm{M}$ & $(10-4) \times 4 \times 3$ & $\begin{array}{c}\text { The crystals were colourless } \\
\text { and highly transparent }\end{array}$ \\
& & a \\
\hline
\end{tabular}

maximum size of the crystal obtained was about $9 \times 4 \times$ $3.6 \mathrm{~mm}^{3}$ as shown in figure 1a. The effect of parameters such as gel density and $\mathrm{pH}$ are summarized in table 1 .

In order to study the effect of $\mathrm{Li}^{+}$ions on the growth and morphology of strontium tartrate crystals, the top solution was mixed with lithium nitrate solution of varying concentrations from $0 \cdot 01-0 \cdot 1 \mathrm{M}$. Tiny crystals appeared within one week at the gel-solution interface and within 12 days below the interface. In the presence of lithium ions, a number of nucleation centres decreased and transparent crystals were formed. The crystals grown at the gelsolution interface were colourless, but those below the interface were slightly pale yellow in colour. The growth of the crystals became stagnant after 20-28 days. The optimum conditions to grow best quality lithium doped strontium tartrate single crystals were at $\mathrm{pH}, 6-6.5$ and gel density, $1.04 \mathrm{~g} / \mathrm{cc}$ for $0.1 \mathrm{M}$ solution of dopant. It was observed that the transparency of the crystals changed with the presence of lithium ion. The presence of $\mathrm{Li}^{+}$ions caused ordering of bound water molecules. Highly polarizing and charged $\mathrm{Li}^{+}$ions cause an overall increase in order in the solution relative to the undoped system. This enhances the diffusion of tartrate ions trapped in the gel cavities to the top solution where the crystals are grown. In this case the crystals were nucleated at the gel surface. These crystals are of bigger size $\left(10 \times 6 \times 4 \mathrm{~mm}^{3}\right)$ than those in the undoped set up and is shown in figure $1 \mathrm{~b}$. The effect of parameters such as gel density and $\mathrm{pH}$ are summarized in table 2 .

\subsection{AES and X-ray diffraction studies}

The atomic spectrum emitted by a sample is used to determine its elemental composition. The wavelength at which emission occurs identifies the element. The dopant concentration of lithium in the grown crystals is $29.8 \mathrm{ppm}$.
Strontium tartrate tetrahydrate crystallizes in the orthorhombic system in the space group $P 2_{1} 2_{1} 2_{1}$ containing four molecules per unit cell with unit cell dimensions, $a=0.948, b=1.096$ and $c=0.946 \mathrm{~nm}$ (Bohandy and Murphy 1968). The lattice parameters of doped crystals are $a=0.946 \mathrm{~nm}, b=1.089 \mathrm{~nm}$ and $c=0.948 \mathrm{~nm}$. There is no appreciable change in the lattice parameters since the concentration of the dopant $\left(\mathrm{Li}^{+}\right)$used in the present study is very low. The XRD pattern of lithium doped strontium tartrate tetrahydrate crystals are shown in figure 2 . The spectrum matches with the data reported in JCPDS file No. 010539 , data of strontium tartrate tetrahydrate crystals.

\subsection{Thermal analysis}

The TGA curve gives useful information regarding the thermal stability and composition of the sample under investigation. The recorded thermogram is first analysed for obtaining the percentage weight loss at different temperatures and hence about the thermal stability and dissociation of the crystals. The thermogravimetric analysis of SrTTH crystals has already been reported (Rahimkutty et al 2001). TGA and DTA curves of pure and lithium doped strontium tartrate tetrahydrate crystals are shown in figures 3 and 4.

Referring to figure $3 \mathrm{a}$, the weight is found to be lost continuously as a function of temperature. By taking the initial weight as standard (wt of the sample taken was $8.0130 \mathrm{mg}$ ), the hydration of these crystals was analysed by comparing it with the molecular weight. The theoretical and observed losses of weight at different temperatures are listed in table 3 . The first stage of decomposition is the dehydration that begins at $398.2 \mathrm{~K}$ and terminates at $507.5 \mathrm{~K}$. The net weight loss of $16.85 \%$ as observed between this temperature range corresponds to the loss of 3 


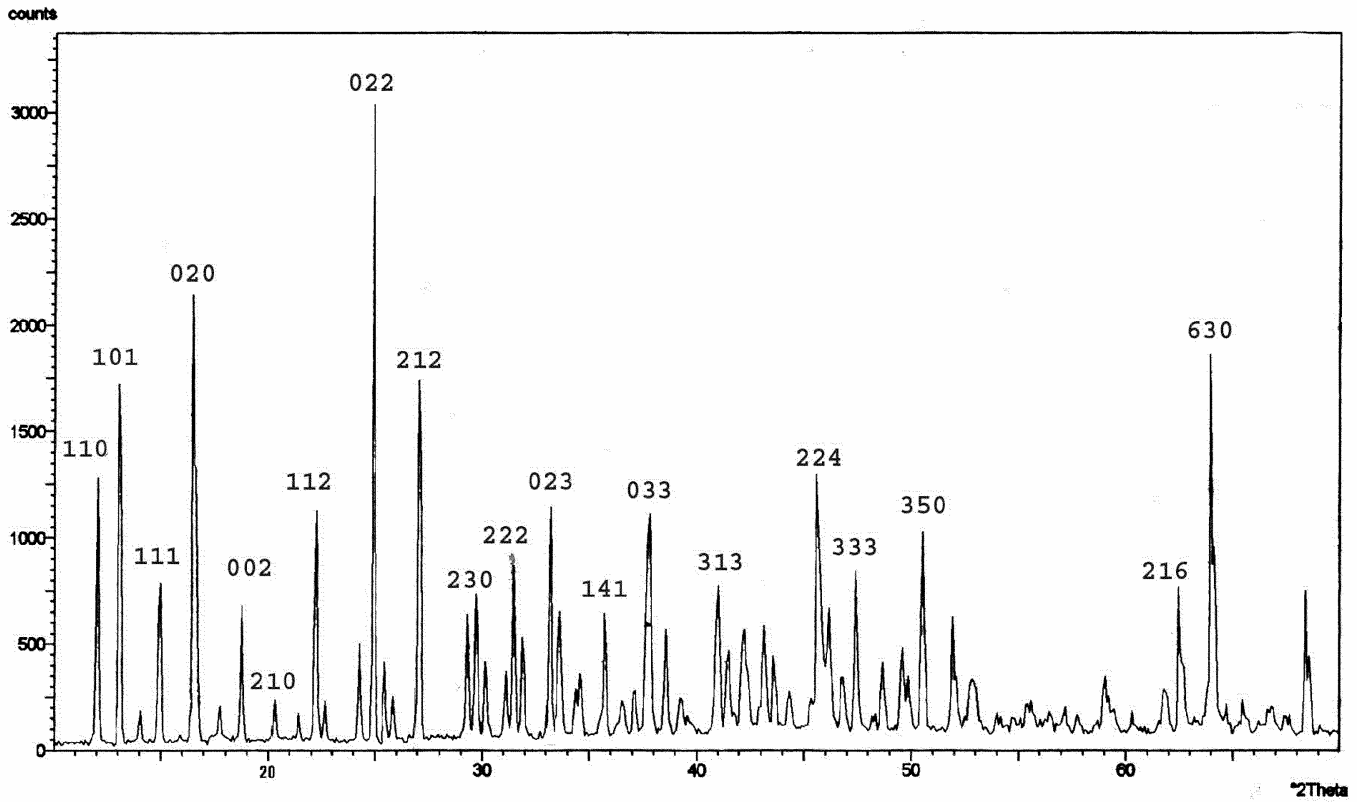

Figure 2. XRD scan of lithium doped SrTTH crystal.

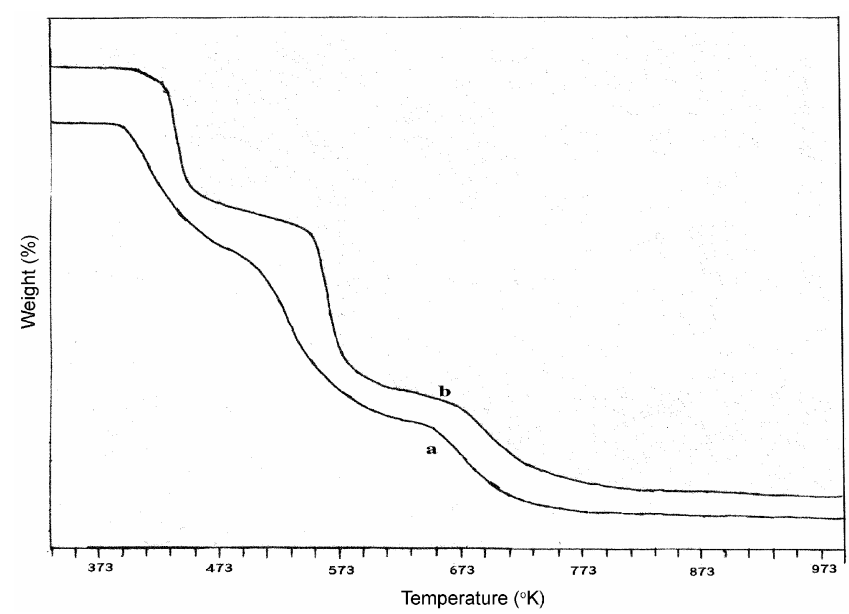

Figure 3. TG curves of a. pure SrTTH and b. lithium doped SrTTH crystals.

water molecules, suggesting the formation of strontium tartrate monohydrate which is stable up to $507.5 \mathrm{~K}$. The second stage of decomposition sets in the crystal at around $507.5 \mathrm{~K}$ and is completed at $640.2 \mathrm{~K}$, resulting in the formation of an intermediate product, viz. strontium oxalate monohydrate complex. This stage of reaction is numerically equal to $20 \cdot 04 \%$. In the third stage of decomposition which seemingly occurs in the temperature interval of $640 \cdot 2-767 \cdot 4 \mathrm{~K}$, the complex strontium oxalate monohydrate eliminates water and decomposes into strontium carbonate $\left(\mathrm{SrCO}_{3}\right)$. This analysis clearly indicates that the grown crystals contain four water molecules. The energy required to dislodge water molecules depends upon how strongly they are locked up in the lattice.

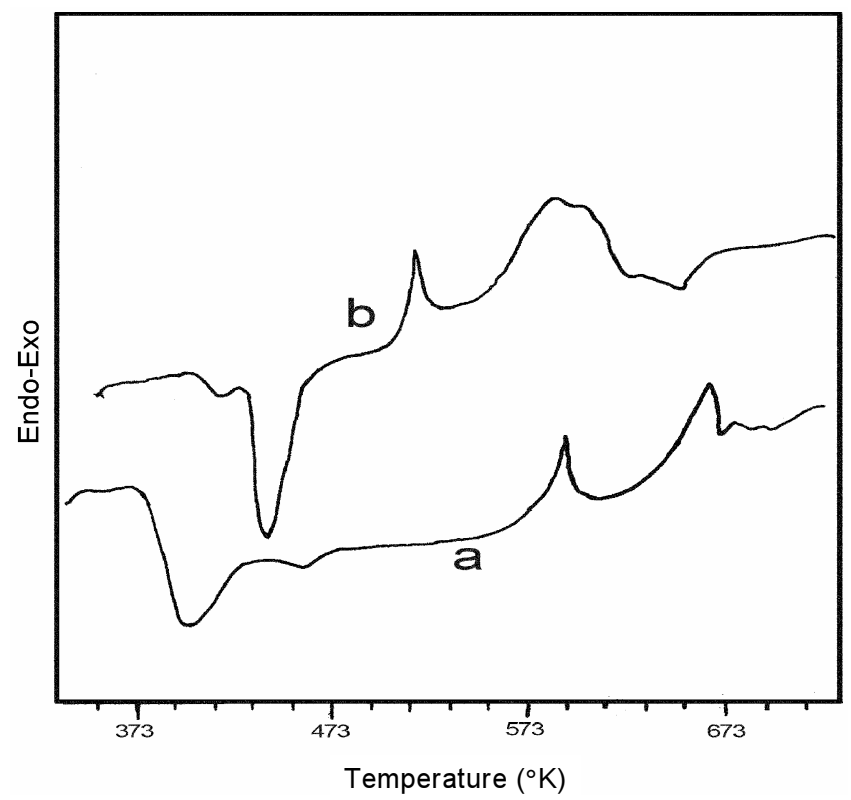

Figure 4. DTA curves of a. pure SrTTH and b. lithium doped SrTTH crystals.

Figure $3 \mathrm{~b}$ shows the TGA curve recorded for lithium doped strontium tartrate tetrahydrate (SrTLiTH). The curve reveals that the decomposition starts at $418.3 \mathrm{~K}$. The elimination of water molecules at a higher temperature for SrTLiTH shows that water molecules are strongly locked in the doped crystal as compared to pure SrTTH crystal. The first stage of decomposition continues up to $553.4 \mathrm{~K}$ leading to the formation of strontium tartrate monohydrate crystals. The second stage starts at $553.4 \mathrm{~K}$ 
Table 3. TGA results of decomposition process of SrTTH and SrTLiTH crystals.

\begin{tabular}{|c|c|c|c|c|c|}
\hline \multirow[b]{2}{*}{ Stage } & \multirow[b]{2}{*}{ Sample } & \multirow[b]{2}{*}{ Temp. range $(\mathrm{K})$} & \multicolumn{2}{|c|}{ Weight loss (\%) } & \multirow[b]{2}{*}{ Reactions } \\
\hline & & & Obsvd. & Calcd. & \\
\hline 1 & $\begin{array}{l}\text { SrTTH } \\
\text { SrTLiTH }\end{array}$ & $\begin{array}{l}398 \cdot 2-507 \cdot 5 \\
418 \cdot 3-553 \cdot 4\end{array}$ & $16 \cdot 85$ & $17 \cdot 55$ & $\mathrm{SrC}_{4} \mathrm{H}_{4} \mathrm{O}_{6} \cdot 4 \mathrm{H}_{2} \mathrm{O}----\mathrm{SrC}_{4} \mathrm{H}_{4} \mathrm{O}_{6} \mathrm{H}_{2} \mathrm{O}$ \\
\hline 2 & $\begin{array}{l}\text { SrTTH } \\
\text { SrTLiTH }\end{array}$ & $\begin{array}{l}507 \cdot 5-640 \cdot 2 \\
553 \cdot 4-663 \cdot 3\end{array}$ & $20 \cdot 04$ & $23 \cdot 65$ & $\mathrm{SrC}_{4} \mathrm{H}_{4} \mathrm{O}_{6} \cdot \mathrm{H}_{2} \mathrm{O}$------ $\mathrm{SrC}_{2} \mathrm{O}_{4} \mathrm{H}_{2} \mathrm{O}$ \\
\hline 3 & $\begin{array}{l}\text { SrTTH } \\
\text { SrTLiTH }\end{array}$ & $\begin{array}{l}640 \cdot 2-767 \cdot 4 \\
663 \cdot 3-783 \cdot 2\end{array}$ & $10 \cdot 75$ & $9 \cdot 32$ & $\mathrm{SrC}_{2} \mathrm{O}_{4} \mathrm{H}_{2} \mathrm{O} \ldots \ldots \ldots \mathrm{SrCO}_{3}$ \\
\hline
\end{tabular}
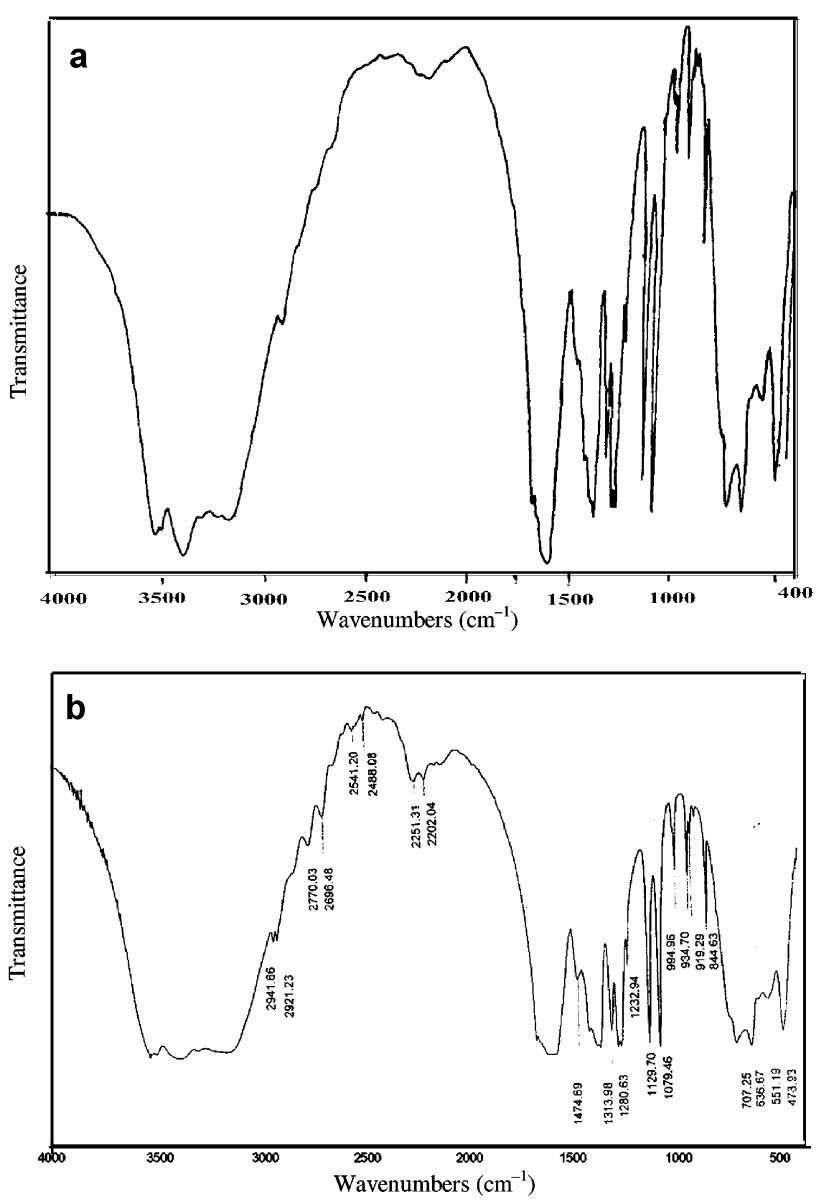

Figure 5. a. FTIR spectrum of pure SrTTH crystal and b. FTIR spectrum of lithium doped SrTTH crystal.

and is completed at $663.3 \mathrm{~K}$ resulting in the formation of strontium oxalate monohydrate crystals. The third stage starts at $663 \cdot 3 \mathrm{~K}$ and terminates at $783.2 \mathrm{~K}$ corresponding to the conversion of oxalate to strontium carbonate. Table 3 gives the summarized results of decomposition of SrTTH and SrTLiTH crystals.

The DTA curves show parallel peaks corresponding to weight losses in TG curves. Figure $4 \mathrm{a}$ illustrates DTA curve recorded for $8.0130 \mathrm{mg}$ of strontium tartrate tetrahydrate crystals. The curve shows an endothermic peak at $402.2 \mathrm{~K}$ corresponding to the elimination of three water molecules. Thermal decomposition reactions are usually endothermic. Figure $4 \mathrm{~b}$ shows DTA curve of lithium doped strontium tartrate tetrahydrate crystal in which the endothermic peak shifted to $435 \cdot 2 \mathrm{~K}$ corresponding to the elimination of three water molecules. No additional peaks are observed other than the peaks corresponding to weight losses in TG curves. So there are no structural changes around the peaks. However, some of the DTA peaks are exothermic. This is due to the concomitant oxidation reaction taking place along with decomposition.

\subsection{FTIR studies}

The FTIR spectra obtained for the grown crystals are shown in figures $5 \mathrm{a}$ and $\mathrm{b}$. A free tartrate ion has two hydroxyl groups. So two bands can be expected for the stretching vibration of the hydroxyl group. A broad band in the region $2900-3900 \mathrm{~cm}^{-1}$ has been observed. This is because the bands due to stretching vibrations of hydroxyl, water and $\mathrm{C}-\mathrm{H}$ groups are overlapping and appear as broader ones. The IR spectra recorded for doped SrTTH crystals were similar to those of pure SrTTH crystals. There was no additional peak due to the presence of dopant. However, the substitution of a $\mathrm{Sr}^{++}$ion (ionic radius $=$ $1.12 \AA$ ) with $2 \mathrm{Li}^{+}$ions (ionic radius $=0.76 \AA$ ) causes the broadening and shifting of these bands. This is a clear indication of presence of the dopants in the crystals. Comparative assignments of bands are furnished in table 4 .

\subsection{Non-linear optical studies}

Kurtz powder SHG experiments (Kurtz and Perry 1968) have been carried out for pure strontium tartrate and lithium doped strontium tartrate. The emission of green radiation from the sample ascertains the SHG property of these two crystals. The amplitude of green radiation was found to increase for SrTLiTH (1.2 times) when compared to pure strontium tartrate crystals. 
Table 4. IR assignments for undoped and lithium doped SrTTH crystals.

\begin{tabular}{|c|c|c|}
\hline Undoped SrTTH $\left(\mathrm{cm}^{-1}\right)$ & Lithium doped SrTTH $\left(\mathrm{cm}^{-1}\right)$ & Assignments \\
\hline $\begin{array}{l}\text { A broad band extending } \\
\text { from } 3200-3550\end{array}$ & $\begin{array}{l}\text { A broad band extending } \\
\text { from } 3050-3510\end{array}$ & Water hydroxyl stretching \\
\hline 2960 & $\begin{array}{l}2941.66 \\
2921.23\end{array}$ & $\mathrm{CH}$-stretching \\
\hline 2250 & $\begin{array}{l}2770 \cdot 03 \\
2696 \cdot 48 \\
2541 \cdot 20 \\
2488 \cdot 08 \\
2251 \cdot 31 \\
2202 \cdot 04\end{array}$ & Combinations and overtone bands \\
\hline $\begin{array}{l}1670 \\
1590\end{array}$ & $1550-1700$ & $\mathrm{C}=\mathrm{O}$ stretch and $\mathrm{H}_{2} \mathrm{O}$ \\
\hline $\begin{array}{l}1470 \\
1450 \\
1382\end{array}$ & $\begin{array}{l}1474 \cdot 69 \\
1452\end{array}$ & $\mathrm{C}=\mathrm{O}$ \\
\hline $\begin{array}{l}1320 \\
1300 \\
1290 \\
1285\end{array}$ & $\begin{array}{l}1313 \cdot 98 \\
1280 \cdot 63 \\
1272 \\
1232 \cdot 94\end{array}$ & In plane and out of $\mathrm{CH}$ bend \\
\hline $\begin{array}{l}1140 \\
1087 \\
1030\end{array}$ & $\begin{array}{l}1129 \cdot 70 \\
1079 \cdot 46\end{array}$ & $\mathrm{C}-\mathrm{O}$ stretch \\
\hline $\begin{array}{r}1010 \\
915 \\
860\end{array}$ & $\begin{array}{l}994.96 \\
934.70 \\
919.29 \\
844.63\end{array}$ & $\mathrm{C}-\mathrm{C}$ stretch \\
\hline $\begin{array}{l}710 \\
660\end{array}$ & $\begin{array}{l}707 \cdot 25 \\
636 \cdot 67\end{array}$ & $\begin{array}{l}\mathrm{H}_{2} \mathrm{O} \text { lib. } \\
\mathrm{CO}_{2} \text { deformation }\end{array}$ \\
\hline $\begin{array}{l}558 \\
475\end{array}$ & $\begin{array}{l}551 \cdot 19 \\
478 \cdot 93\end{array}$ & $\begin{array}{l}\text { Torsion } \mathrm{COO} \text { and torsion } \\
\mathrm{COOH}\end{array}$ \\
\hline
\end{tabular}

\section{Conclusions}

There has been always a keen and competitive race to grow perfect single crystals with purity, perfection and transparency. Good quality single crystals of pure SrTTH $\left(9 \times 4 \times 3.6 \mathrm{~mm}^{3}\right)$ and lithium doped strontium tartrate tetrahydrate $\left(10 \times 6 \times 4 \mathrm{~mm}^{3}\right)$ are grown by gel method. Observations show that lithium ion doping enhances the size and transparency of the crystals, since lithium ion acts as a 'structure maker' (Wright 1988). TGA analysis revealed that doped crystals are found to be thermally more stable than pure crystals. The FTIR spectrum of the doped crystals indicates the formation of chemical bonds due to the dopant. Crystal structure of the compound was confirmed by X-ray diffractometry and dopant concentration with AES system. Kurtz and Perry powder technique measurement shows that the second harmonic efficiency of the doped crystal increases. The major defect of gel inclusion into the grown crystals can be avoided in this method of growth, where crystals are grown over the surface of the gel. Another advantage is that bigger crystals suitable for device applications can be grown by this solution-gel technique.

\section{Acknowledgements}

Authors express their thanks to KSCSTE, Govt. of Kerala, for financial support and STIC, Cochin, for providing analytical facilities. One of the authors (BSK) also thanks UGC for awarding a teacher fellowship.

\section{References}

Arora S K, Patel V, Kothari A and Amin B 2004a Crystal Growth and Design 4343

Arora S K, Patel V, Amin B and Kothari A 2004b Bull. Mater. Sci. 27141

Arora S K, Patel V and Kothari A 2004c Mater. Chem. Phys. 84 323

Arora S K, Patel V, Chudasama B and Amin B 2005a J. Cryst. Growth 275 e657

Arora S K, Patel V, Kothari A and Chudasama B 2005b J. Am. Ceram. Soc. 883469

Bohandy J and Murphy J C 1968 Acta Crystallogr. B24 286

Dennis J and Henisch H K 1967 J. Electrochem. Soc. 114263

Henisch H K 1973 Crystal growth in gels (University Park, Ohio: The Pennsylvania State University Press) 
Kurtz S K and Perry T T 1968 J. Appl. Phys. 393798

Patel A R and Arora S K 1976 J. Mater. Sci. 11843

Patel A R and Arora S K 1977 J. Mater. Sci. 122124

Rahimkutty M H, Rajendra Babu K, Sreedharan Pillai K, Sudarsana Kumar M R and Nair C M K 2001 Bull. Mater. Sci. 24249

Rajendra Babu K, Deepa M, Nair C M K and Vaidyan V K 1997 Cryst. Res. Technol. 32733

Rethinam F J, Arivuoli D, Ramasamy S and Ramasamy P 1993 Cryst. Res. Technol. 28861
Rethinam F J, Arivuoli D, Ramasamy S and Ramasamy P 1994a Mater. Res. Bull. 29309

Rethinam F J, Ramasamy S, Arivuoli D and Ramasamy P 1994b J. Mater. Sci. Lett. 13263

Satyanarayana N, Hariharan K and Radhakrishna S $1985 \mathrm{~J}$. Mater. Sci. 201993

Viji R K, Gowri B and Sahaya Shajan X 2005 Indian J. Phys. 791373

Wright M R 1988 Nature of electrolyte solutions (Macmillan Education) p. 103 